\title{
Review of the Phytoneering Research \& Experience Summit (PRES) 2019 "building bridges between nature's healing potential and evidence-based medicine - 20 years of phytoneering"
}

\author{
Kurt G. Naber ${ }^{1 *}$ (D), Oleg Apolikhin², Vladimir Kozlov ${ }^{3}$ and David W. Kennedy ${ }^{4}$
}

\begin{abstract}
This brief communication summarizes the key highlights presented at the 2019 Phytoneering Research and Experience Summit held at the Palma Convention Centre and the educational finca Sa Canova (Majorca, Spain) from the 25th to the 27th of July 2019.

More than 100 professionals from academia, industry and public services took part. The scientific programme included three dedicated symposium sessions and a moderated poster session. In total, 13 oral communications and 23 posters were presented.

The invite-only conference, entitled: "Phytoneering Research \& Experience Summit (PRES) 2019 - Building bridges between nature's healing potential and evidence-based medicine - 20 years of phytoneering", had participants from 20 different nations, reflecting the international character of the event. This participant group included researchers as well as clinicians with different fields of interest, e.g. gynaecology, urology, respiratory diseases and paediatrics. They all share an interest in evidence-based phytotherapy and phytoneering, which is the combination of ancient knowledge about medicinal plants and state-of-the-art technology to produce evidence-based herbal medicine. This approach offers solutions to reduce the growing number of unnecessarily prescribed antibiotics in the abovementioned areas, with the aim of preventing further progression of antibiotic resistance. The conference was sponsored by Bionorica ${ }^{\circledR}$ SE.
\end{abstract}

Keywords: Phytoneering, Urology, Gynaecology, Respiratory, Spain

\section{Gynaecological session}

Two well-investigated plant extracts for the treatment of premenstrual syndrome and climacteric complaints (Prof. Wolfgang Wuttke, Germany)

Symptoms of premenstrual syndrome (PMS) and the climacteric period are caused by disorders of the endocrinal system. Herbal medicines can be a very effective and safe alternative to hormonal therapy. In his presentation, Prof. Wuttke presented two well-studied evidence-based plant extracts for the treatment of PMS and menopausal

\footnotetext{
* Correspondence: kurt@nabers.de

1 Department of Urology, Technical University of Munich, Munich, Germany Full list of author information is available at the end of the article
}

symptoms. PMS involves the dopaminergic system of the brain, which regulates the release of pituitary prolactin and is also involved in premenstrual mastodynia and emotional imbalance. A special extract of Vitex agnuscastus (VAC), BNO 1095, has shown to effectively alleviate symptoms of PMS and cycle-related breast pain (mastodynia). Diterpenes, especially, contribute to the efficacy with their dopaminergic properties. The production of the high-quality special extract BNO 1095 is done with a unique patented method, providing diterpenes of the labdane and/or clerodane type as particularly effective types. With respect to their dopaminergic activity, clerodienoles were determined as being the 
most active components of VAC special extract BNO 1095 in these studies [1].

Hot flashes are the most common menopausal complaint reported, which may occur up to 30 times a day. A common result of hot flashes is sweating. Night sweats in particular disturb restorative sleep. It is known that low concentrations of neurotransmitters are the cause of these symptoms, especially hot flashes. Serotonin-, GABA-, and dopamine-agonists have been isolated from a special extract of the rhizome of Cimicifuga racemosa $(\mathrm{CR})$. In several clinical studies, the special extract CR BNO 1055 extract shows good efficacy, leading to a significant reduction of hot flashes by up to $80 \%$. The plant extract BNO 1055 acts in places such as the central nervous system, in which the temperatureregulating centre controls the occurrence of hot flashes. Studies also revealed an excellent safety profile of BNO 1055, which offers an alternative treatment option to conjugated oestrogens, which are used during hormone replacement therapy (HRT) and have been discussed to have associated serious risks [2].

\section{Metabolic aspects of dyshormonal diseases of the female reproductive system. Potentials for phytotherapy (Dr. Tetiana Struk, Ukraine)}

Currently there is a significant increase in the prevalence of overweight and metabolic syndrome in the world. By 2025 , about $50 \%$ of women and $40 \%$ of men are expected to be overweight. In addition, with the modern lifestyle, there is an increase in effects of chronic stress and, as a result, a significant increase in diseases associated with impaired emotional and mental health. These two pathological conditions underline the majority of dyshormonal diseases of the female reproductive system. Hyperprolactinemia due to chronic stress and overweight are the leading pathogenetic mechanisms leading to dyshormonal disorders. Special preparations of VitexAgnus Castus (Mastodynon (BNO 1020 and BNO 1035), Cyclodynon ${ }^{\circ}$ (BNO 1095) have a multi-vector effect that allows several mechanisms of the development of dyshormonal diseases to be eliminated at once: they eliminate hyperprolactinemia and estrogen-progesterone imbalance, and also eliminate the effects of chronic stress on the organism; have an anti-anxiety effect (comparable efficacy with fluoxetine for treatment of premenstrual dysphoric disorders) and lead to a decrease in urine cortisol levels, which indicates a beneficial effect of VAC at metabolic level. Thus, the use of standardized phytopreparations on the basis of VAC can be considered an important component in treating dyshormonal reproductive system diseases in women who are overweight, with metabolic syndrome and an increased level of stress tension.

\section{Urological session}

The urological session was chaired by Prof. Naber and Prof. Apolikhin.

\section{The clinical significance of BNO 1045 (Canephron ${ }^{\otimes} \mathrm{N}$ ) in the treatment of patients with urolithiasis (Prof. Vadim Rudenko, Russia)}

Urolithiasis is one of the most common urological diseases, affecting around $1-3 \%$ of the population worldwide. In developed countries, such as the US, the incidence is even higher. $7 \%$ of men and $3 \%$ of women suffer from this condition, thus accounting for $20-40 \%$ of the total contingent of patients in urological hospitals. The condition has a recurrent nature - after 3 years, the risk of relapse is around $40 \%$.

The optimal treatment ensures the effective and safe evacuation of stone fragments and the reduction of residual fragments, as they cause recurrent stone formation. Lithokinetic therapy, also known as medical expulsive therapy (MET) is one of the treatment options used to facilitate the spontaneous passage of ureteral stones. Typical medications consist of either antispasmodics, alpha-blockers, non-steroidal anti-inflammatory drugs (NSAIDs) or a combination of these. Herbal preparations, especially those with a combination of herbs resulting in a broad multi-target action (e.g. diuretic, anti-inflammatory and spasmolytic effects), are widely used in the context of urinary tract diseases. In addition to the aforementioned multifaceted nature of effects, herbal medication often results in a lower incidence of adverse reactions compared to synthetic drugs, especially during long-term use.

Due to the multi-substance nature and high complexity of herbal drugs, low quality or the lack of standardization is a potential issue. Clinical efficacy determined in GCP-controlled clinical trials can only be transferred to the same product/herb if the same standardized process can be assured. Prof. Rudenko stated that Bionorica -as the inventor of the phytoneering principle - is a manufacturer that is using the most modern scientific methods and technologies to ensure the highest quality and standardization.

One of Bionorica's blockbuster products is BNO 1045 $\left(\right.$ Canephron $\left.^{\circ} \mathrm{N}\right)$, a herbal drug with complex action consisting of rosemary leaves, centaury herb and lovage root (RCL) in the ratio 1:1:1. BNO 1045 has outstanding success in the treatment and prophylaxis of acute and recurring urinary tract infections and evidence in the treatment of urinary calculi.

The objective of the presented study [3] was to demonstrate that a combination of modern technologies (extracorporeal shockwave therapy (ESWL), percutaneous nephrolithotomy (PCNL) and ureteroscopy (URS)) in combination with phytotherapy allows the effective 
treatment of various clinical forms of urolithiasis (kidney stone disease (KSD)). After extracorporeal shockwave lithotripsy (ESWL), lithokinetic therapy (herbal diuretics, antispasmodics, $\alpha$-blockers, NSAIDs) accelerates the discharge of stone fragments, increases the frequency of complete removal of stone fragments and reduces the need for additional pain relief. The main group included 80 patients who were prescribed BNO 10452 capsules 3 times a day after ESWL (Dornier Gemini). The control group included 59 patients who underwent standard antispasmodic therapy after ESWL (the groups were comparable in localization and size). Clinical efficacy criteria were the frequency of renal colic; duration and percent of stone fragments discharge (stone-free rate (SFR)); change of blood and urine laboratory parameters, daily diuresis and urine $\mathrm{pH}$, as well as individual drug tolerance. For the analysis with regard to metabolic disorders, patients of both groups were divided into the following subgroups (main group with BNO 1045 - control group): patients with hypercalciuria (31.2-30.5\%); patients with hyperuricemia and hyperuricuria (26.2$27.1 \%$ ); patients with hypercalciuria and hyperuricemia (22.5-23.7\%); and patients with hyperphosphaturia (20.0-18.6\%), respectively. Treatment with BNO 1045 provided a significant decrease in leukocyturia (leukocyturia was observed in only $10 \%$ of patients), as well as the normalization of urine $\mathrm{pH}$ in the range of 6.2-6.8 and an increase in daily diuresis up to $2500 \mathrm{ml}$. In the control group, leukocyturia persisted in $57.6 \%$ of patients, while urine $\mathrm{pH}$ and daily diuresis remained almost unchanged. During the first 5 days, stone discharge was noted in $62(77.5 \%)$ patients in the main group, and in 24 patients $(40.7 \%)$ in the control group. In the main group, the level of pain was rated 5 points according to the visual analogue scale (VAS), and renal colic was observed in $16(20 \%)$ patients. In the control group, the level of pain was 7 points according to the VAS, and renal colic was noted in $25(42.4 \%)$ patients. In a comprehensive study of stone fragments, it was found that calcium-oxalate and mixed types of stone predominated in both groups.

In patients receiving $\mathrm{BNO}$ 1045, after 1.5 months of treatment, urinary calcium had already decreased by $50 \%$ in $31.2 \%$ of patients; a decrease in urinary uric acid and an increase in urine $\mathrm{pH}$ was noted in $26.2 \%$ of patients, and a normalization of urinary calcium and serum uric acid with normalization of urine $\mathrm{pH}$ was recorded in $42.5 \%$ of patients. However, for patients in the control group, a positive change in the parameters due to the treatment used was recorded only after 3 months.

Prof. Rudenko summarized the effects of BNO 1045 as follows: the stone-free rate (SFR) after ESWL was increased while the pain intensity was reduced. A decrease in leukocyturia, an increase in daily diuresis and a normalization/stabilization of urine $\mathrm{pH}$ were observed. A reduction in the concentration of uric acid in the blood and urine and a reduction of hypercalciuria were achieved. BNO 1045 substantially prevented stone formation, reduced the risk of relapse and increased the time to occurrence. A high tolerability makes the drug suitable for long-term use in the context of lithokinetic therapy and stone formation metaphylaxis.

A recently published study [4] showed that the use of BNO 1045, as a means of preventing stone formation, is pathogenetically substantiated and can reduce the risk of relapse, as well as increase the time to its occurrence.

\section{Bacteria and host interaction in UTI with new perspectives on non-antibiotic treatments, (Prof. Björn Wullt, Sweden)}

UTI are among the most common bacterial infections in women, which can lead to bothersome symptoms in the lower or upper urinary tract. Most frequently, bacterial establishment results in an asymptomatic carrier state without causing symptoms or disease, and even protects against symptomatic infection [5]. Whether the outcome of bacterial establishment results in symptomatic disease or asymptomatic carriage depends on both the host susceptibility and the virulence of the strain. With regard to uropathogenic E. coli (UPEC), several virulence factors were described, such as adhesive fimbriae, toxins affecting the function of the host cells, capsules preventing attacks by host defence molecules, iron binding proteins and immune inhibitors. Virulent strains activate innate immune signalling, leading to the secretion of chemokines and cytokines and the subsequent recruitment of inflammatory cells. Asymptomatic bacteriuria (ABU) strains, however, do not express or are even devoid of these factors [6]. In his presentation, Prof. Wullt focused on the host reaction to UTI, such as Toll-like receptors (TLRs), especially the TLR4. TLRs are key players in the innate immune system [7], especially in the defence mechanisms that accompany urinary tract infections (UTIs).

\section{Paradigm shift in the treatment of acute, uncomplicated urinary tract infections: non-antibiotic therapy options (Prof. Florian Wagenlehner, Germany)}

"Can symptomatic treatment for uncomplicated cystitis be an alternative to antibiotic treatment?" This question was raised by Prof. Wagenlehner during his talk. Antibiotic therapy has clearly demonstrated its superiority over placebo treatment, especially with regard to microbiological eradication at end of treatment (OR 10.67) and after end of treatment (OR 5.38) [8]. Thus, antibiotic therapy has a high significance in cystitis and is recommended by various guidelines, such as the German S3 Guideline [9]. On the other hand, collateral damage, 
epidemiologic side effects of antibiotics and the severe impact of typical antibiotics on the microbiome are increasingly reported [10]. Several studies with pain medication (ibuprofen and diclofenac, respectively) versus antibiotics (fosfomycin, pivmecillinam and norfloxacin, respectively) came to the conclusion that symptomatic therapy with NSAIDs alone was successful [11], however, the symptoms resolved faster under antibiotic therapy.

Can phytotherapy be an alternative in the treatment of acute, uncomplicated cystitis? The combination phytotherapy with lovage, rosemary and centaury (BNO 1045, Canephron ${ }^{\circ}$ N) has been extensively evaluated in preclinical settings and in early clinical studies with promising results. In a randomised, double-blind, phase III non-inferiority clinical trial, the combination phytotherapy BNO 1045 was compared for 7 days with a single dose of fosfomycin trometamol (FT) in the treatment of acute lower uncomplicated urinary tract infections (uUTIs) [12]. The primary endpoint was the proportion of patients who received additional antibiotics (ABs) to treat uUTIs between days 1 and 38. Between days 1 and 38, $238(83.5 \%)$ patients in the BNO 1045 group and $272(89.8 \%)$ patients in the FT group received no additional antibiotics. At a $15 \%$ non-inferiority margin, BNO 1045 was non-inferior to FT in treating acute, lower uUTIs. For the first time, the validated Acute Cystitis Symptom Score (ACSS) was used as a self-reporting questionnaire for the diagnosis of acute cystitis and for the assessment of the symptom score during follow-up visits. The secondary endpoint of the study was the decrease in symptoms, which was nearly overlapping in both study arms. Overall, this clinical study demonstrated that symptomatic treatment with an evidence-based phytotherapeutical can be a viable alternative to antibiotics for the treatment of acute lower uUTIs and can potentially help to reduce the use of antibiotics and antibiotic resistance.

\section{Respiratory session}

The respiratory session was chaired by Prof. Kozlov and Prof. Kennedy.

\section{Sinupret ${ }^{\circledR}$ extract: a history of success (Prof. Claus Bachert, Belgium)}

In Bionorica's long history spanning more than 85 years, Sinupret ${ }^{\circ}$ has been a part of the portfolio since the beginning. Although the combination of medicinal plants like gentian, primrose, common sorrel, elder and vervain has remained unchanged, Sinupret ${ }^{\circ}$ has been constantly developed and improved over the years. The latest addition to the Sinupret ${ }^{\circ}$ brand was Sinupret ${ }^{\circ}$ extract in 2012, using cutting-edge extraction techniques to increase the content of active ingredients without changing the size of the tablet. In his presentation, Prof. Bachert highlighted milestones of Sinupret's success story, including preclinical pharmacological data and high-quality clinical studies he personally conducted with his colleagues $[11,12]$. All of this made it possible to develop Sinupret ${ }^{\circ}$ from an experience-based remedy into an evidence-based phytomedicine, recommended in several national and international rhinosinusitis guidelines.

\section{The protective effect of BNO 1010 (Sinupret ${ }^{\oplus}$ oral drops) on the ciliated epithelium in rhinitis. (Prof. Svitlana Yaremchuk, Ukraine)}

The objective of the presented work was to study the effect of BNO 1010 on nasal mucosa atrophic changes in a rat model of experimental rhinitis. The study was designed as a preclinical (in-vivo), open, controlled (in parallel groups) study. Sixty female Wistar rats were allocated for the experimental study. To study the effect of the phytopreparation on atrophy of the nasal epithelium, the animals were divided into 3 groups. The 1st group $(n=20)$ was without experimental acute rhinitis. The 2nd group $(n=20)$ was with experimental acute rhinitis and without treatment with BNO 1010. The 3rd group $(n=20)$ was with experimental acute rhinitis and treatment with BNO 1010. Experimental rhinitis was induced by placing rats in a swimming pool with cold water, followed by a draught of cool air. The effect of oral BNO 1010 treatment on histopathological changes in nasal mucosa was assessed 3 and 14 days post challenge. The results were analysed using a normal or modified Student's t-test for independent groups. On day 3 , the height of the olfactory epithelium $[\mu \mathrm{m}]$ in the BNO 1010 group was 23\% above the rhinitis (without BNO 1010) group and a cross-sectional area of goblet cells $[\mu \mathrm{m} 2]$ in the BNO 1010 group was $16 \%$ higher than in the group without treatment. On day 14, the height of the olfactory epithelium $[\mu \mathrm{m}]$ in the BNO 1010 group was $18 \%$ above the rhinitis (without BNO 1010) group.

In conclusion, the histological evaluation demonstrated that BNO 1010 applied during acute rhinitis attenuate atrophic and destructive changes to the ciliated epithelium. Oral administration of the herbal medicinal product BNO 1010 weakens atrophy of the nasal mucosal ciliated epithelium in a rat model of acute rhinitis [13].

\section{Guidelines of the German Respiratory Society for the diagnosis and treatment of adults suffering from acute, subacute and chronic cough 2019. (Dr. Peter Kardos, Germany)}

Symptoms of cough can be provoked by many different causes, such as respiratory tract infections, asthma, COPD, reflux or even certain heart conditions. Based on 
the disease underlying the symptoms, a cough can be classified as an acute or chronic cough. In his presentation, Dr. Kardos gave an overview about how chronic and acute cough are diagnosed and summarized the most relevant treatment options, including phytotherapy. The fixed combinations thyme/ivy and thyme/primrose are recommended in acute cough by the most current cough guideline published by the German Respiratory Society in early 2019. Dr. Kardos is the primary author of this guideline [14].

Comparative study of the therapeutic efficacy, safety and tolerability of BNO 1030 (Imupret ${ }^{\circledR}$ ) in patients with acute non-bacterial tonsillitis. (Prof. Vasil Popovych, Ukraine)

Prof. Popovych presented his research on the treatment of non-bacterial tonsillitis with BNO 1030 as an add-on to the standard treatment compared to standard treatment only in children and adolescents aged 6-18 years. One hundred eighteen patients (the main group) were treated with BNO 1030 in addition to the standard treatment (Benzydamine hydrochloride + antipyretics if necessary), while 120 patients (the control group) were treated with the standard treatment only. The criteria for the evaluation of efficacy were the reduction in the severity of symptoms, as a part of the Local Tonsillitis Manifestation Scale [LTMS (0-4 points per symptom): hyperaemia of the posterior wall; hyperaemia of the tonsils; swelling, plaque; pain (swallowing); pain (rest); throat irritation; cough]; responders / non-responders ratio (regression of the sum of the severity points by 75\%); reduced need for NSAIDs / antipyretics; antibiotics; "therapeutic gain" in days. The criterion used to evaluate safety was the number of patients with adverse events.

Supplemental use of a phytoneering medicinal product BNO 1030 for treatment of acute tonsillitis has been shown to promote considerable decrease of tonsillitis clinical symptoms, improved assessment of patients' general condition and their quality of life, decrease of the rate of use of antipyretics and overall treatment duration without any adverse events. Prospects for further studies consist in investigation of anti-relapse efficacy of the drug in patients with recurrent tonsillitis [15].

\section{Effectiveness and tolerability of BNO 1030/1035 (Tonsilgon ${ }^{\circledast} \mathrm{N}$ ) in the treatment of recurrent upper respiratory tract infections in children: a non- interventional study in Russia. (Prof. Vera Vavilova, Russia)}

Acute respiratory viral infections (ARVI) in children are common and often recurrent. Since the treatment of most acute respiratory viral infections does not require the use of antibiotics, excessive antibiotic use should be avoided, and herbal medicinal products can be regarded as an effective treatment alternative. The purpose of the study was to demonstrate the efficacy and tolerability of the combined herbal medicinal product BNO 1030/1035 in children aged 2-11 years in Russia.

The study design involved 518 children with ARVI (2013-2014), who were included in a prospective noninterventional study in 14 Russian research centres. Before inclusion in the study, patients had at least 2 episodes of acute respiratory viral infections registered during the last 6 months. On day 1 (= visit 1) BNO $1030 / 1035$ was prescribed as dragees or oral drops at an age-adjusted dosage. The duration of treatment was approximately 14 days (day $15=$ visit 2 ) followed by a 30 day follow-up period (day 45). The efficacy of therapy was assessed by evaluating objective and subjective symptoms and the frequency of a positive response to therapy. The time until cessation of symptoms, compliance, use of concomitant medications, and adverse reactions were also evaluated.

Results of the study showed a similar distribution of patients by age and sex. The three most common diagnoses at inclusion were rhinopharyngitis, pharyngitis and tonsillitis. Objective symptoms for these pathologies (hyperaemia of the mucous membrane and swelling of the tonsils) were completely eliminated on visit 2 in 93.4-97.9\% of patients. Most of the subjective symptoms were eliminated within 4 days, while the typical symptomatic phase was 7 days for all past ARVI episodes. In general, the time to symptom relief was reduced by 3 days, with $99.5 \%$ of patients responding to the treatment. Good or very good tolerability of the herbal medicinal product was reported for $97 \%$ of patients. Overall compliance was high $(88.2 \%)$. This leads to the conclusion that BNO 1030/1035 is a safe and effective option for the treatment of acute respiratory viral infections in children (aged 2-11 years) which reduces the duration of ARVI symptoms [16].

\section{Strategies to avoid unnecessary antibiotics: how evidence-based tools can help us in our daily routine. (Dr. Michael Hubmann, Germany)}

Antimicrobial resistances (AMRs) are growing from year to year, making antibiotics increasingly ineffective. The WHO estimates the annual number of deaths attributed to AMRs at around 10 million by 2050, far more than cancer or road traffic accidents. A large number of antibiotics are still prescribed for respiratory tract infections such as coughing, rhinosinusitis or tonsillitis. This certainly contradicts the recommendations in most relevant guidelines for respiratory tract infections. Strategies for delayed prescription of antibiotics could provide a valid solution to this dilemma. Dr. Hubmann gave an insight into his daily routine as a paediatrician and presented strategies to avoid unnecessary antibiotics. His approach 
to addressing this issue requires a high level of communication between the attending practitioner and the patient, as well as the use of symptomatic treatment options, such as evidence-based phytotherapy.

\section{Conclusion}

This event showed once more the importance of evidence-based phytomedicine as an alternative to widely used standard therapy options such as antibiotics or hormone replacement therapy. Such alternatives are urgently needed, especially in respiratory tract infections (RTIs), where, despite the recommendations in all common guidelines, the majority of antibiotics for systemic use in primary care is prescribed for both adults and children. Several studies have revealed that the patientdoctor relationship and poor communication between them plays an important role in the unnecessary prescription of antibiotics [17]. Considering that $57 \%$ of the European population is unaware that antibiotics are ineffective against viruses causing RTIs, and $44 \%$ do not know that antibiotics have no effect against self-limiting diseases such as the common cold or influenza, this leads to a high rate of unnecessary prescription of antibiotics for RTIs, ranging from 50 to $90 \%$ [17]. During PRES, several international experts presented studies demonstrating that phytomedicines provide an alternative therapeutic option in many different RTIs, such as rhinosinusitis, bronchitis and tonsillitis with good symptom control, high patient satisfaction and with a good safety profile.

Evidence-based phytotherapy also offers a promising alternative to antibiotic therapies in rather benign diseases like acute, uncomplicated cystitis. Nowadays, guidelines also mention the possibility of a purely symptom-based therapy for uncomplicated acute lower UTIs. The study presented, with a fixed combination of lovage, centaury and rosemary (BNO 1045), revealed the non-inferiority of the combination when compared to a commonly used antibiotic in uncomplicated UTIs, offering further treatment options aside from antibiotics.

In conclusion, it is fair to say that evidence-based phytotherapy provides an alternative to chemical-synthetic drugs in various therapeutic areas. Especially with regard to the threat of increasing antimicrobial resistance, evidence-based herbal therapy and phytoneering could help to reduce the prescription of unnecessary antibiotics.

\section{Acknowledgements}

Bionorica SE, Neumarkt, Germany for their organizational support of the summit.

\section{Authors' contributions}

KGN had the lead in writing the manuscript. All authors read and approved the final manuscript.
Funding

The organization of PRES was funded by Bionorica SE.

Availability of data and materials

Not applicable.

Ethics approval and consent to participate

Not applicable.

Consent for publication

Not applicable.

\section{Competing interests}

KGN reports personal fees from Adamed, Apogepha, Aristo, Biomerieux, Bionorica SE, Daiichi Sankyo, Enteris Biopharma, Eumedica, GlaxoSmithKline, Gruenenthal Mexico, Helperby Therapeutics, Hermes, Medice, Meiji Seika Pharma, MerLion, MSD Sharp \& Dohme, OM Pharma and Vifor, Paratek, Roche, Rosen Pharma, and Zambon.

\section{Author details}

'Department of Urology, Technical University of Munich, Munich, Germany. ${ }^{2} \mathrm{~N}$. Lopatkin Scientific Research Institute of Urology, Ministry of Health of the Russian Federation, Moscow, Russia. ${ }^{3}$ ENT Department, Central State Medical Academy, Moscow, Russia. ${ }^{4}$ Perelman School of Medicine, University of Pennsylvania, Philadelphia, USA.

Received: 31 October 2019 Accepted: 29 January 2020

Published online: 17 February 2020

\section{References}

1. Wuttke W, Seidlová-Wuttke D, Gorkow C. The Cimicifuga preparation BNO 1055 vs. conjugated estrogens in a double-blind placebo-controlled study: effects on menopause symptoms and bone markers. Maturitas. 2003; 44(Supplement):S67-77.

2. Collaborative Group on Hormonal Factors in Breast Cancer. Type and timing of menopausal hormone therapy and breast cancer risk: individual participant meta-analysis of the worldwide epidemiological evidence. Lancet. 2019. https://doi.org/10.1016/S0140-6736(19)31709-X.

3. Alyaev YG, Sayenko VS, Rudenko VI, Dzeranov NK, Amosov AV. Phytogenic drug Canephron ${ }^{\otimes} \mathrm{N}$ in the treatment of patients with urolithiasis. Urologiia. 2012;(6) https://urologyjournal.ru/en/archive/article/11563.

4. Demidko YL, Rudenko VI, Grigoryan VA, Enikeev ME, Allenov SN, Kasiteridi IG, Ujegov TA, Amosov AV. Clinical value of Canephron N after surgical treatment of urinary stone disease. Urologiia. 2019;2:15-20.

5. Wullt B, Sundén F, Grabe M. Asymptomatic Bacteriuria is harmless and even protective: don't treat if you don't have a very specific reason. Eur Urol Focus. 2018;5. https://doi.org/10.1016/j.euf.2018.07.004.

6. Ragnarsdóttir B, Svanborg C. Pediatr Nephrol. 2012;27:2017. https://doi.org/ 10.1007/s00467-011-2089-1.

7. Engel D, Dobrindt U, Tittel A, et al. Tumor necrosis factor alpha- and inducible nitric oxide synthase-producing dendritic cells are rapidly recruited to the bladder in urinary tract infection but are dispensable for bacterial clearance. Infect Immun. 2006;74(11):6100-7. https://doi.org/10. 1128/IAl.00881-06

8. Falagas ME, Kotsantis IK, Vouloumanou EK, Rafailidis PI. Antibiotics versus placebo in the treatment of women with uncomplicated cystitis: a metaanalysis of randomized contolled trials. J Infect. 2009;58(2):91-102.

9. Kranz J, Schmidt S, Lebert C, Schneidewind L, Mandraka F, Kunze M, Helbig S, Vahlensieck W, Naber K, Hoyme U, Schmiemann G, Wagenlehner FM. The 2017 update of the German clinical guideline on epidemiology, diagnostics, therapy, prevention, and management of uncomplicated urinary tract infections in adult patients: part II: therapy and prevention. Urol Int. 2018; 100(3):271-8.

10. Naber $\mathrm{KG}$, et al. How the microbiome is influenced by the therapy of urological diseases: standard versus alternative approaches. Clin Phytosci. 2017:3:8.

11. Naber KG, Tandogdu Z, Köves B, Bonkat G, Wagenlehner F. UTI - quo vadis? New alternatives to treat uncomplicated urinary tract infections. Clin Phytosci. 2019;5:40. https://doi.org/10.1186/s40816-019-0132-0.

12. Wagenlehner FM, Abramov-Sommariva D, Höller M, Steindl H, Naber KG. Non-antibiotic herbal therapy (BNO 1045) versus antibiotic therapy 
(Fosfomycin Trometamol) for the treatment of acute lower uncomplicated urinary tract infections in women: a double-blind, parallel-group, randomized, multicentre, non-inferiority phase III trial. Urol Int. 2018;101: 327-36. https://doi.org/10.1159/000493368 Jund R, Mondigler M, Steindl H, Stammer H, Stierna P, Bachert C; ARhiSi II study group. Clinical efficacy of a dry extract of five herbal drugs in acute viral rhinosinusitis. Rhinology. 2012; 50(4):417-26. doi: 10.4193/Rhino12.015.

13. Yaremchuk S, Zabolotny D, Vareniuk I, Makarchuk N, Veselsky S. Sinupret ${ }^{\oplus}$ oral drops protect against respiratory epithelium atrophy in experimental acute rhinitis. Clin Phytosci. 2015;1:8. https://doi.org/10.1186/s40816-0150009-9.

14. Kardos P, Dinh QT, Fuchs KH, Gillissen A, Klimek L, Koehler M, Sitter $H$, Worth H. Guidelines of the German Respiratory Society for diagnosis and treatment of adults suffering from acute, subacute and chronic cough. Pneumologie. 2019;73(3):143-80. https://doi.org/10.1055/a-0808-7409 Epub 2019 Feb 18.

15. Popovych V, Koshel I, Malofiichuk A, Pyletska L, Semeniuk A, Filippova O, Orlovska R. A randomized, open-label, multicenter, comparative study of therapeutic efficacy, safety and tolerability of BNO 1030 extract, containing marshmallow root, chamomile flowers, horsetail herb, walnut leaves, yarrow herb, oak bark, dandelion herb in the treatment of acute non-bacterial tonsillitis in children aged 6 to 18 years. Am J Otolaryngol. 2019;40(2):26573. https://doi.org/10.1016/j.amjoto.2018.10.012 Epub 2018 Oct 24.

16. Vavilova VP, Abramov-Sommariva D, Steindl H, Wonnemann M, Ryzhova EG, Rusova TV, Lebedenko AA, Kolchenko IV. Effectiveness and tolerability of Tonsilgon ${ }^{\oplus} \mathrm{N}$ in the treatment of recurrent upper respiratory tract infections in children: a non-interventional study in Russia. Clin Phytosci. 2016;2:6. https://doi.org/10.1186/s40816-016-0020-9.

17. Machowska A, Stålsby LC. Drivers of irrational use of antibiotics in Europe. Int J Environ Res Public Health. 2018;16(1). https://doi.org/10.3390/ ijerph16010027.

\section{Publisher's Note}

Springer Nature remains neutral with regard to jurisdictional claims in published maps and institutional affiliations.

\section{Submit your manuscript to a SpringerOpen ${ }^{\circ}$ journal and benefit from:}

- Convenient online submission

- Rigorous peer review

- Open access: articles freely available online

- High visibility within the field

- Retaining the copyright to your article

Submit your next manuscript at $\boldsymbol{\nabla}$ springeropen.com 